\title{
Controlling Quantum Transport via Dissipation Engineering
}

\author{
François Damanet๑, ${ }^{1, *}$ Eduardo Mascarenhas, ${ }^{1}$ David Pekker, ${ }^{2,3}$ and Andrew J. Daley ${ }^{1}$ \\ ${ }^{1}$ Department of Physics and SUPA, University of Strathclyde, Glasgow G4 ONG, United Kingdom \\ ${ }^{2}$ Department of Physics and Astronomy, University of Pittsburgh, Pittsburgh, Pennsylvania 15260, USA \\ ${ }^{3}$ Pittsburgh Quantum Institute, Pittsburgh, Pennsylvania 15260, USA
}

(Received 18 April 2019; published 31 October 2019)

\begin{abstract}
Inspired by the microscopic control over dissipative processes in quantum optics and cold atoms, we develop an open-system framework to study dissipative control of transport in strongly interacting fermionic systems, relevant for both solid-state and cold-atom experiments. We show how subgap currents exhibiting multiple Andreev reflections - the stimulated transport of electrons in the presence of Cooper pairs - can be controlled via engineering of superconducting leads or superfluid atomic gases. Our approach incorporates dissipation within the channel, which is naturally occurring and can be engineered in cold gas experiments. This opens opportunities for engineering many phenomena with transport in strongly interacting systems. As examples, we consider particle loss and dephasing, and note different behavior for currents with different microscopic origin. We also show how to induce nonreciprocal electron and Cooperpair currents.
\end{abstract}

DOI: 10.1103/PhysRevLett.123.180402

Introduction.-Understanding and controlling the outof-equilibrium dynamics of strongly interacting many-body systems is at the forefront of research in quantum physics across a variety of subfields in experiment and theory. In this context, opportunities to achieve their control via dissipation mechanisms have arisen [1,2], as is applied for few-body systems in quantum optics [3,4]. This is especially true in cold-atom platforms, where large separations between frequency scales allow well-controlled theoretical models and implementations of dissipative processes, as realized for laser cooling and trapping [5]. The longer timescales of cold-atom experiments also allow dynamics to be tracked and potentially controlled time dependently [6,7]. Out-of-equilibrium transport dynamics remain a ubiquitous paradigm in the solid state [8], and recent developments in cold-atom systems have also made it possible to engineer quantized transport of atoms between reservoirs, as well as quantum point contacts and waveguides [9-12]. Here we explore the emerging new opportunity of using dissipation engineering to achieve control of quantum transport properties, that are relevant for both cold-atom and solid-state platforms.

We study transport in a system of strongly interacting fermions coupled to weakly interacting reservoirs, as can be realized with cold atoms using optical tweezers connecting larger superfluids, or with solid-state devices using quantum dots (QDs) coupled to superconducting leads (S). In a traditional S-QD-S tunneling junction, subgap transport is known to be suppressed for weak electron tunneling as compared to the gap of the attached leads [13]. Here we demonstrate that subgap transport can be recovered even in the regime of weak tunneling. This is done via reservoir engineering that allows for independent control of Cooperpair and single-electron channels. Such channel separation can be accomplished in the solid state by adding two largegap superconductors to a traditional S-QD-S junction, producing a four-terminal structure, or in cold atoms considering driving from a molecular Bose-Einstein condensate [14].

Subgap currents in this context are produced by multiple Andreev reflections (MARs) [13,15-17], i.e., stimulated transport of electrons via exchange of Cooper pairs. MARs have been observed in the solid state [18-21] and cold atoms [11], and their signatures can be used to reveal topological phase transitions related to Majorana bound states formation [22]. We show how to engineer wellresolved MAR peaks under weak electron tunneling, and show how these behave in the presence of dissipation in the channel-providing a diagnostic tool for the microscopic nature of the current. We also show that for asymmetric coupling the reciprocity of the engineered system is broken, yielding electron and Cooper-pair currents dependent on the bias direction. This represents a genuinely new way of generating nonreciprocal transport of electrons and Cooper pairs.

We investigate the transport properties of the junction with an open-system approach, while most of the theoretical works rely on Keldish nonequilibrium Green functions or scattering techniques. These approaches are able to treat the tunneling rate $\gamma$ between the QD and the leads nonperturbatively, but usually treat the Coulomb interaction $U$ between the QD electrons perturbatively or within a mean-field treatment [23-26]. In contrast, open-system approaches such as input-output theories [27-30] or master 
equations [31-33] work well in the opposite regime: for arbitrary interaction $U$ but weak tunneling rate $\gamma$, implying that MARs have been left beyond their scope. In our framework, the large-gap superconducting leads behave effectively as time-dependent coherent drives of Cooper pairs on the QD (analogous to laser fields in quantum optics). This dynamical model is naturally cast as a dissipative Floquet system, for which we derive a Floquet-Born-Markov master equation [34-37] capturing MARs up to arbitrary order. Our open-system framework provides an opportunity to study the effects of controlled or uncontrolled dissipation acting on the QD. We thus analyze the response of the currents to fermion losses and dephasing, and show, in particular, robustness of the currents against dephasing. We use in the following natural units in which $\hbar=k_{B}=e=1$, where $-e$ is the electron charge.

Model.-To represent the separate control of Cooper-pair driving, we consider a four-terminal QD connected to two pairs of left $(L)$ and right $(R)$ superconducting leads by tunnel junctions, as depicted in Fig. 1. In each pair, we consider one lead in the single-particle mean-field description with a moderate energy gap $\Delta_{\ell}(\ell=L, R)$, and one described only by its condensed fraction of Cooper pairs, assuming that the gap is so large that single-particle excitations are irrelevant. A bias voltage $V=V_{L}-V_{R}$ is generated between the pairs of superconductors, where $V_{L}$ and $V_{R}$ are the voltages of each side. The QD Hamiltonian reads

$$
H_{\mathrm{QD}}=\sum_{s=\downarrow, \uparrow} \omega c_{s}^{\dagger} c_{s}+U c_{\uparrow}^{\dagger} c_{\uparrow} c_{\downarrow}^{\dagger} c_{\downarrow},
$$

and describes electrons of spin $s$, energy $\omega$, and Coulomb interaction $U$. The $\mathrm{QD}$ is an effective four-level system spanned by the nonoccupied, single-occupied, and doubleoccupied states $\{|0\rangle,|\downarrow\rangle,|\uparrow\rangle,|\downarrow \uparrow\rangle\}$. The coupling of the QD to the large-gap superconducting leads (red superconductors in Fig. 1) gives rise to a pairing of the QD electrons, i.e., the proximity effect [17], and results in an effective time-dependent QD Hamiltonian of the form

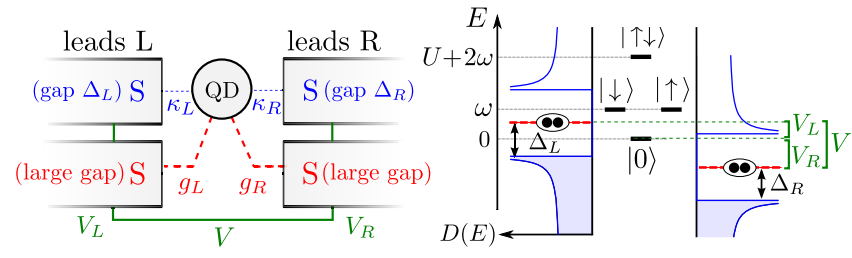

FIG. 1. Left: Sketch of the four-terminal QD tunneling junction with Cooper-pair and electron tunneling of amplitudes $g_{\ell}$ and $\kappa_{\ell}$ $(\ell=L, R)$. Right: Corresponding energy diagram. The moderategap superconducting leads are characterized by Bogoliubov quasiparticles of density of states $D(E)$ (blue). The large-gap superconducting leads are only characterized by their Cooper-pair condensates (red). The applied bias voltage $V_{\ell}$ is the same for the moderate- and large-gap leads.

$$
H_{\mathrm{QD}}^{\mathrm{eff}}(t)=H_{\mathrm{QD}}+\sum_{\ell=L, R}\left(g_{\ell} e^{2 i V_{\ell} t} c_{\downarrow} c_{\uparrow}+\text { H.c. }\right),
$$

where $g_{\ell}$ is the Cooper-pair tunneling amplitude between the QD and the large-gap superconducting $\ell=L, R$. Hence, the coupling of the large-gap superconductors with the QD takes the form of a driving of the transition between the nonoccupied and double-occupied states $|0\rangle$ and $|\downarrow \uparrow\rangle$ of the QD.

We obtain the dissipative dynamics of the QD by coupling the Hamiltonian (2) to the superconductors with moderate gaps $\Delta_{\ell}$ (blue superconductors in Fig. 1) under an open-system approach, by deriving a Floquet-BornMarkov master equation [34-37] for the QD. The leads, considered in a mean-field single-particle description, act as baths of Bogoliubov quasiparticles of density of states $D_{\ell}(E) \propto \Theta\left(|E|-\Delta_{\ell}\right)|E| /\left(\sqrt{E^{2}-\Delta_{\ell}^{2}}\right)$. The tunneling of electrons between the leads and the QD is described by a standard tunneling Hamiltonian of the form $H_{\text {int }}=$ $\sum_{\ell} \kappa_{\ell} \sum_{k s}\left(b_{\ell k s}^{\dagger} c_{s}+\right.$ H.c. $)$, where $\kappa_{\ell}$ is the electron tunneling amplitude and $b_{\ell k s}$ the annihilation operator of an electron of spin $s$ and momentum $k$ in the moderate-gap lead $\ell$. The derivation of the master equation, in second order in $H_{\text {int }}$, results in a single-particle tunneling rate $\gamma_{\ell} \propto \kappa_{\ell}^{2}$ (the typical linewidths of the QD levels) considered as the smallest parameter. Note that while treating perturbatively the single-particle coupling, the master equation describes the QD Coulomb interaction $U$ exactly, as in Refs. $[32,33]$. See Supplemental Material for details of the derivation [38].

Engineering of transport.-From the solutions of the master equation, we calculate the particle current in the leads as a function of the applied bias voltages, taken as opposite from each other for the sake of simplicity $\left(V_{L}=-V_{R}=V / 2\right)$. Figure 2 shows the particle current $I$ in both the moderate- and large-gap right leads as a function of $V$. We consider the electron-hole symmetric case $\omega=-U / 2$, and vanishing or not Cooper-pair tunneling $g \equiv g_{\ell}$, taken here real and identical for left and right leads. We also consider identical single-particle tunneling rates $\gamma \equiv \gamma_{\ell}$ between the QD and the moderate-gap superconducting leads.

When $g=0$, the large-gap superconducting leads are disconnected from the QD, and our system simply consists in a conventional S-QD-S tunneling junction [32,33]. Only one peak of current is observed [see Fig. 2(a)], whose shape is related to the superconductor density of states $D_{\ell}(E)$. The peak appears when $E_{i}>\omega>E_{f}$, where $E_{i}$ is the energy of the highest occupied state of the left lead and $E_{f}$ is the energy of the lowest nonoccupied state of the right lead (see energy diagram I in Fig. 2). For high bias, the particle current tends to the value $2 \gamma$ of normal leads. For low bias $\left(\gamma \ll V<4 \Delta_{\ell}\right)$, i.e., in the subgap region (where no resonance between left-lead occupied and right-lead 


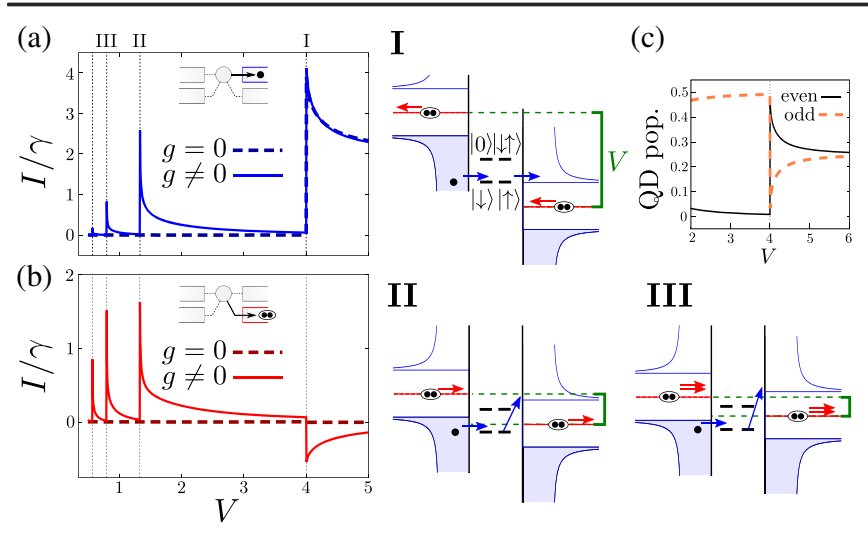

FIG. 2. Particle current $I$ (in units of $\gamma$ ) in the right moderategap lead (a) and large-gap lead (b) as a function of the bias voltage $V$ for $g=g_{\ell}=0$ (dashed line) and 0.5 (solid line). Other parameters are $U=2, \omega=-1, \gamma=10^{-2}$, and $T_{\ell}=0$, in units chosen so that $\Delta_{\ell} \equiv \Delta=1$. Subgap currents via MARs appear for nonvanishing $g$. (I)-(III) Energy diagrams corresponding to the standard resonant tunneling (I) and the first and second MARs (II) and (III). (c) Steady states QD averaged populations for states with even $(|0\rangle,|\downarrow \uparrow\rangle$, black line) and odd $(|\downarrow\rangle,|\uparrow\rangle$, dashed orange line) number of electrons.

nonoccupied states exist), no current is observed as a result of the weak-coupling approximation. Indeed, for $\gamma \ll \Delta$, Andreev reflection at the interface with the moderate-gap superconductor is negligible.

Connecting the large-gap superconducting leads to the QD (i.e., setting $g \neq 0$ ) allows Andreev reflections to occur. Under such process, an electron (hole) is reflected as a hole (electron) producing the emission (absorption) of a Cooper pair in the large-gap superconducting leads [see Fig. 2(b)]. After some reflections, electrons of the QD acquire enough energy to tunnel into the moderate-gap superconducting lead. This produces well-resolved single-particle subgap currents more and more pronounced as $g$ increases. These processes are represented in our Floquet-Born-Markov formalism by decay channels corresponding to QD transition (quasi)energies shifted by multiple of Cooper-pair energies (see Supplemental Material [38]). The subgap currents are located at $V=2(|\omega|+\Delta) /(2 n+1)$, where $n=1,2, \ldots$ denotes the $n$th MAR (see energy diagrams II and III in Fig. 2 corresponding, respectively, to the first and second Andreev reflections). This can be obtained from the condition $E_{i}+n V=\omega=E_{f}-n V$, in which $n$ denotes the number of Cooper-pairs transfer from the left to the right lead. Note, however, that in general the bias voltage at which a MAR peak appears is a function of both the QD charging energy $U$ and $\omega$ (see Supplemental Material [38]). Hence, while the tunneling between the QD and the moderate-gap leads is always sequential due to the weak-coupling regime (one electron per time), it can be assisted by transfer of an arbitrary number of Cooper pairs between the large-gap superconductors, thanks to the stronger tunneling amplitude $g$. This represents reservoir engineering of subgap single-particle currents. This is our first important result. Interestingly, the Cooper-pair current in the right large-gap superconducting lead is negative outside the subgap region. We attribute this phenomenon to a supercurrent (i.e., Cooper-pair current) reversal, due to the modification of parity of the QD when the voltage exceeds the value delimiting the subgap border, as can be seen in Fig. 2(c) $[13,43]$. Note that the sign and amplitude of the supercurrent are dependent of the phases of the superconductors (not shown).

Effects of particle loss and dephasing.-In the previous section, we showed that dissipation induced by reservoir engineering can be used to control subgap transport. Here we examine the robustness of the produced subgap currents against the presence of incoherent processes, which are inherent in real experimental setups. We incorporate these effects into our master equation through an additional dissipator of the form $\mathcal{D}_{I}(\rho)=\gamma_{I}\left(2 L \rho L^{\dagger}-\left\{L^{\dagger} L, \rho\right\}\right)$, where $\gamma_{I}$ is the rate of the incoherent process and $L$ the corresponding Lindblad operator (see Supplemental Material [38]). For cold-atoms experiments, the dissipation in the channel is often in this Markovian form as can be derived from first principles [2].

We first consider the effects of particle loss (i.e., $\gamma_{I} \equiv \gamma_{\text {loss }}$, $L=c_{s}$ ) acting on the QD. This occurs naturally in the coldatom platforms through background gas collisions, and could be engineered using electron beams [44] or light scattering quantum gas microscopes with single-site resolution [45-47] (analogous to x-ray scattering in the solid state). In Figs. 3(a)-3(d), we show the particle currents in all the leads as a function of the bias voltage for increasing loss rates $\gamma_{\text {loss. }}$. The presence of losses results in competing effects. On the one hand, the additional decay channel tends to empty the QD faster. This results in an increase (decrease) of the currents of electrons entering (reaching) the moderategap superconducting leads. On the other hand, pushing the QD towards the nonoccupied state $|0\rangle$ increases the effects of the driving (since the driving only affects the QD in the nonoccupied or double-occupied states), which favors MARs and thus raises subgap currents. Hence, while source currents [Figs. 3(a) and 3(c)] only increase due to electrons losses, drain currents [Figs. 3(b) and 3(d)] are subjected to these competing effects, exhibiting amplitude increase or decrease depending on the voltage bias.

We then consider the effects of dephasing (i.e., $\gamma_{I} \equiv \gamma_{\text {deph }}$, $L=c_{s}^{\dagger} c_{s}$ ) acting on the QD, which occurs naturally through coupling to additional degrees of freedom in the solid state, and can be engineered in cold atoms through light scattering or noise [2,48-51]. We show that dephasing acting on the QD affects identically the source and drain Cooper-pair currents, whereas it leaves unchanged the electron currents. Figure 3(e) shows the current of Cooper pair leaving the QD to reach the right large-gap superconductor for different dephasing rate $\gamma_{\mathrm{deph}}$. Our results show that increasing the dephasing rate reduces 

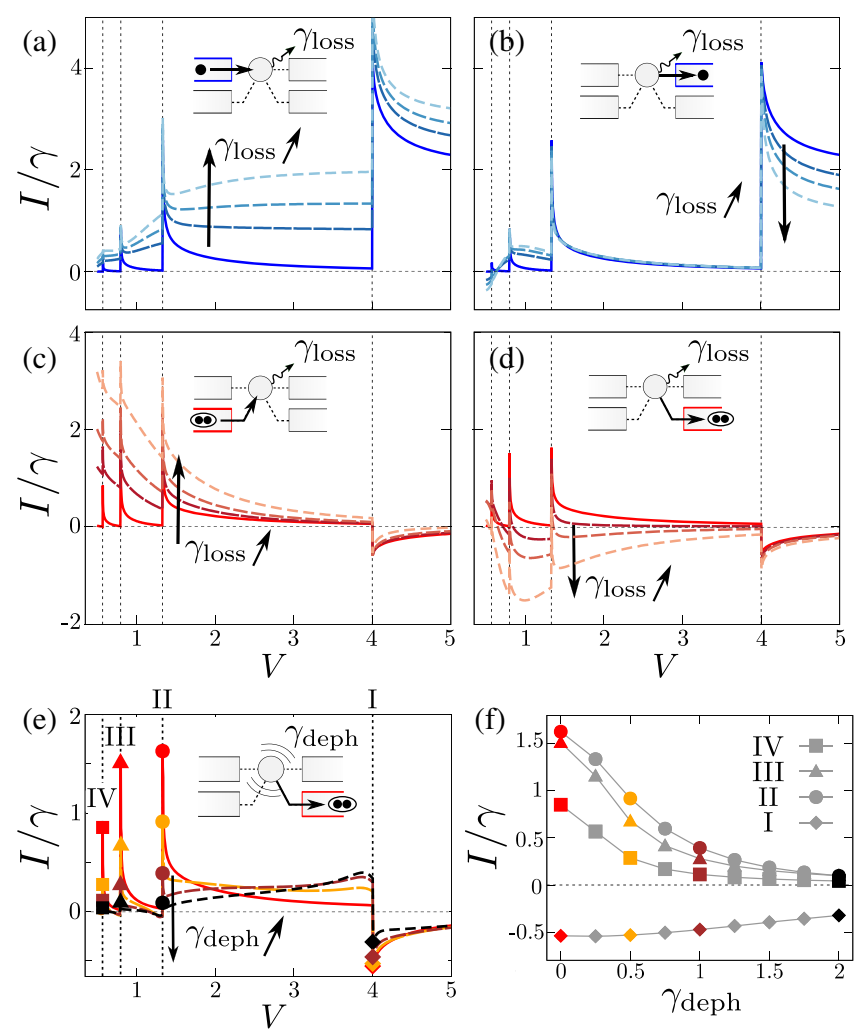

FIG. 3. Current-voltage characteristics under the effects of electron loss (a)-(d) and dephasing (e),(f) acting on the QD. Currents of electrons entering (a) and leaving (b) the QD and of Cooper pairs entering (c) and leaving (d) the QD as a function of the bias voltage $V$ for different electron loss rate $\gamma_{\text {loss }}$ (from solid to dashed lines, $\left.\gamma_{\text {loss }}=0,0.5 \gamma, \gamma, 2 \gamma\right)$. Other parameters as above. Current of Cooper pairs in the right large-gap superconducting lead as a function of $V$ for $\gamma_{\mathrm{deph}}=0,0.5,1$, and 2 (e), and as a function of $\gamma_{\mathrm{deph}}$ for $V=2(|\omega|+\Delta) /(2 n+1)$ with $n=0,1,2$, and 3 corresponding to the peaks I, II, III, and IV (f). Other parameters as above.

the size of the subgap peaks [see Fig. 3(f)]. This can be understood as a consequence of the blurring of the QD energy levels caused by the dephasing. Hence, dephasing tends to destroy Cooper-pair subgap currents, but does not affect the single-particle currents. This suggests these latter are robust against phonon (photon) scattering in condensed matter (cold-atomic) systems.

Nonreciprocal subgap transport.-Finally, we show how to generate nonreciprocal subgap transport. Biasdirection-dependent properties are generally a desired feature of nanoscale devices, and are known to result from the presence of asymmetry and nonlinearity. Nonreciprocal transport at the quantum level has been investigated in spin [52-60] and QD systems [61-65]. This includes the paradigmatic Pauli blockade effects in a double-QD junction, where a nonreciprocal electron current has been observed for asymmetric QD energy levels [62]. For a
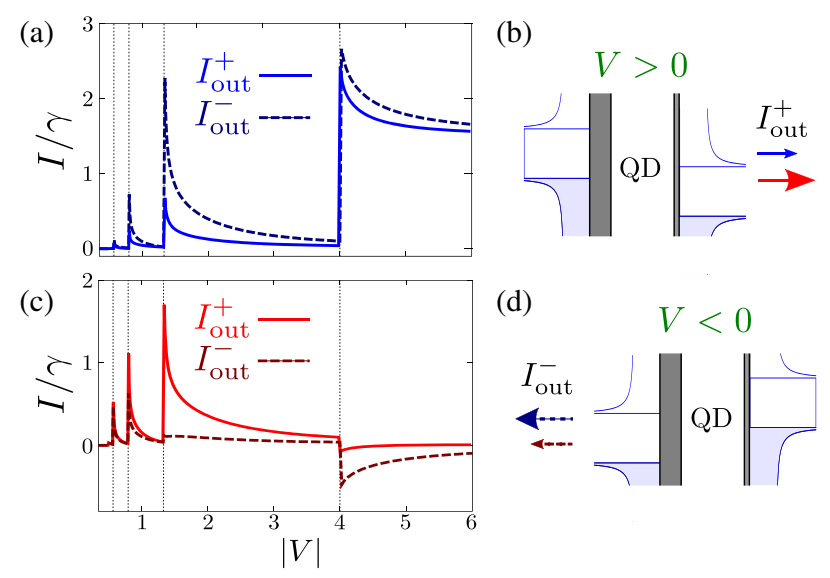

FIG. 4. Current-voltage characteristics in the moderate-gap (a) and large-gap (c) superconducting leads for asymmetric singleparticle tunneling rate $\gamma_{L}=3 \gamma_{R}=1.5 \times 10^{-2}$. In both plots, the solid (dashed) lines curves correspond to the current for positive [negative] bias voltage $V$, as depicted in the diagram (b) [(d)] on the right. Other parameters are $U=2, \omega=-1, \gamma=10^{-2}$, $T_{\ell}=0$, and $g_{\ell}=0.5$, in units of $\Delta_{\ell} \equiv \Delta$.

single $\mathrm{QD}$, the required asymmetry can be provided by different left and right tunneling rates. In a S-QD-S junction, in the intermediate coupling regime $\left(\gamma_{\ell} \sim \Delta_{\ell}\right)$, nonreciprocal conductance has been observed and explained as originating from asymmetric Kondo resonance at the contact with the leads [66]. Here we show that for asymmetric weak single-particle tunneling rates $\gamma_{L} \neq \gamma_{R}$, the reciprocity of the transport properties can be broken as soon as the Cooper-pair tunneling amplitudes $g_{\ell}$ are nonzero. In Fig. 4, we plot the current-voltage characteristics for the moderate- [Fig. 4(a)] and large-gap [Fig. 4(c)] superconducting leads for positive and negative bias voltage [see Figs. 4(b) and 4(d)] for $\gamma_{L}=3 \gamma_{R}$. While the total current (the sum of electron and Cooper-pair currents) is still reciprocal (not shown), its electron and Cooper-pair contributions become dependent on the bias direction, as can be clearly seen in Fig. 4. In particular, the current of electrons (Cooper pair) is larger (smaller) for negative (positive) bias. We interpret this phenomenon as a Cooperpair-assisted nonreciprocal transport, since it occurs only for nonzero $g_{\ell}$. Indeed, for $g_{\ell}=0$, the electron current is reciprocal (not shown). We believe it is a genuinely new way of breaking reciprocity, since while keeping reciprocal the total current, its electron and Cooper-pair contributionswhich could be measured independently in our four-terminal scheme-become asymmetric.

Conclusion.-We developed a quantum-optics-inspired framework to study the dynamics of strongly interacting fermions in tunneling junctions under the influence of dissipation and driving, relevant for both solid-state and cold-atom platforms. For concreteness, we studied the dynamics of a QD coupled to superconducting leads in a four-terminal configuration, where two large-gap 
superconducting leads are added to a traditional S-QD-S tunneling junction. We demonstrate the possibility of controlling subgap transport via dissipation engineering. We showed that the added leads generate subgap transport based on MARs despite weak electron tunneling and studied the effects of electron loss and dephasing acting on the QD. Finally, we showed that the Cooper-pair driving provided by the added leads is a new way of breaking the reciprocity of the junction, generating nonreciprocal electron and Cooper-pair subgap currents based on MARs.

Our results could be investigated in both solid-state and cold-atom experiments. They could be generalized to multi-QD tunneling junctions and to include the presence of measurement and feedback loop to control the transport dynamics of fermions in tunneling junctions [67]. More possible outlooks include reservoir engineering of (Floquet-)Majorana fermions [14,68,69], or studies of the interplay between dissipation and driving in thermodynamics problems such as thermoelectric effects [70] or quantum heat engines [71] involving superconductors.

All data underpinning this publication are openly available from the University of Strathclyde KnowledgeBase at [72]

Work at the University of Strathclyde was supported by the EPSRC Programme Grant DesOEQ (EP/P009565/1), and by the EOARD via AFOSR Grant No. FA9550-18-10064. Work at the University of Pittsburgh was supported by NSF PIRE-1743717.

*Corresponding author francois.damanet@strath.ac.uk

[1] M. Müller, S. Diehl, G. Pupillo, and P. Zoller, Engineered open systems and quantum simulations with atoms and ions, Adv. At. Mol. Opt. Phys. 61, 1 (2012).

[2] A. J. Daley, Quantum trajectories and open many-body quantum systems, Adv. Phys. 63, 77 (2014).

[3] H. M. Wiseman and G. J. Milburn, Quantum Measurement and Control (Cambridge University Press, Cambridge, England, 2009).

[4] C. Gardiner and P. Zoller, The Quantum World of UltraCold Atoms and Light Book II: The Physics of QuantumOptical Devices, 1st ed. (Imperial College Press, London, 2015).

[5] H. J. Metcalf and P. var der Straten, Laser Cooling and Trapping (Springer, Berlin, 2001).

[6] I. Bloch, J. Dalibard, and W. Zwerger, Many-body physics with ultracold gases, Rev. Mod. Phys. 80, 885 (2008).

[7] I. Bloch, J. Dalibard, and S. Nascimbène, Quantum simulations with ultracold quantum gases, Nat. Phys. 8, 267 (2012).

[8] C. W. J. Beenakker and H. Van Houten, Quantum transport in semiconductor nanostructures, Solid State Phys. 44, 1 (1991).

[9] S. Krinner, D. Stadler, D. Husmann, J.-P. Brantut, and T. Esslinger, Observation of quantized conductance in neutral matter, Nature (London) 517, 64 (2015).
[10] S. Krinner, T. Esslinger, and J.-P. Brantut, Two-terminal transport measurements with cold atoms, J. Phys. Condens. Matter 29, 343003 (2017).

[11] D. Husmann, S. Uchino, S. Krinner, M. Lebrat, T. Giamarchi, T. Esslinger, and J.-P. Brantut, Connecting strongly correlated superfluids by a quantum point contact, Science 350, 1498 (2015).

[12] M. Lebrat, P. Grišins, D. Husmann, S. Häusler, L. Corman, T. Giamarchi, J.-P. Brantut, and T. Esslinger, Band and Correlated Insulators of Cold Fermions in a Mesoscopic Lattice, Phys. Rev. X 8, 011053 (2018).

[13] S. De Franceschi, L. Kouwenhoven, C. Schönenberger, and W. Wernsdorfer, Hybrid superconductor-quantum dot devices, Nat. Nanotechnol. 5, 703 (2010).

[14] L. Jiang, T. Kitagawa, J. Alicea, A. R. Akhmerov, D. Pekker, G. Refael, J. I. Cirac, E. Demler, M. D. Lukin, and P. Zoller, Majorana Fermions in Equilibrium and in Driven Cold-Atom Quantum Wires, Phys. Rev. Lett. 106, 220402 (2011).

[15] A. Andreev, The Thermal Conductivity of the Intermediate State in Superconductors, Sov. Phys. JETP 19, 1228 (1964).

[16] C. Beenakker, Random-matrix theory of quantum transport, Rev. Mod. Phys. 69, 731 (1997).

[17] A. Martin-Rodero and A. Levy Yeyati, Josephson and Andreev transport through quantum dots, Adv. Phys. 60, 899 (2011).

[18] M. Octavio, M. Tinkham, G. E. Blonder, and T. M. Klapwijk, Subharmonic energy-gap structure in superconducting constriction, Phys. Rev. B 27, 6739 (1983).

[19] J-D. Pillet, C. H. L. Quay, P. Morfin, C. Bena, A. Levy Yeyati, and P. Joyez, Andreev bound states in supercurrent-carrying carbon nanotubes revealed, Nat. Phys. 6, 965 (2010).

[20] M. R. Buitelaar, W. Belzig, T. Nussbaumer, B. Babic, C. Bruder, and C. Schönenberger, Multiple Andreev Reflections in a Carbon Nanotube Quantum Dot, Phys. Rev. Lett. 91, 057005 (2003).

[21] T. Dirks, T. L. Hughes, S. Lal, B. Uchoa, Y.-F. Chen, C. Chialvo, P. M. Goldbart, and N. Mason, Transport through Andreev bound states in a graphene quantum dot, Nat. Phys. 7, 386 (2011).

[22] P. San-Jose, J. Cayao, E. Prada, and R. Aguado, Multiple Andreev reflection and critical current in topological superconducting nanowire junctions, New J. Phys. 15, 075019 (2013).

[23] A. Levy Yeyati, J. C. Cuevas, A. López-Dávalos, and A. Martin-Rodero, Resonant tunneling through a small quantum dot coupled to superconducting leads, Phys. Rev. B 55, R6137 (1997).

[24] A. Zazunov, R. Egger, C. Mora, and T. Martin, Superconducting transport through a vibrating molecule, Phys. Rev. B 73, 214501 (2006).

[25] L. Dell' Anna, A. Zazunov, and R. Egger, Superconducting nonequilibrium transport through a weakly interacting quantum dot, Phys. Rev. B 77, 104525 (2008).

[26] K. Kang, Transport through an interacting quantum dot coupled to two superconducting leads, Phys. Rev. B 57, 11891 (1998).

[27] C. P. Search, S. Pötting, W. Zhang, and P. Meystre, Inputoutput theory for fermions in an atom cavity, Phys. Rev. A 66, 043616 (2002). 
[28] C. W. Gardiner, Input and output in damped quantum systems III: Formulation of damped systems driven by fermions fields, Opt. Commun. 243, 57 (2004).

[29] C. W. Gardiner and P. Zoller, Quantum Noise, 3rd ed. (Springer, Berlin, 2004).

[30] N. Zhao, J.-L. Zhu, R.-B. Liu, and C. P. Sun, Quantum noise theory for quantum transport through nanostructures, New J. Phys. 13, 013005 (2011).

[31] H.-P. Breuer and F. Petruccione, The Theory of Open Quantum Systems (Oxford University Press, Oxford, 2006).

[32] D. S. Kosov, T. Prosen, and B. Žunkovič, A Markovian kinetic equation approach to electron transport through a quantum dot coupled to superconducting leads, J. Phys. Condens. Matter 25, 075702 (2013).

[33] S. Pfaller, A. Donarini, and M. Grifoni, Subgap features due to quasiparticle tunneling in quantum dots coupled to superconducting leads, Phys. Rev. B 87, 155439 (2013).

[34] Y. Yan, Z. Lü, and H. Zheng, Resonance fluorescence of strongly driven two-level system coupled to multiple dissipative reservoirs, Ann. Phys. (Amsterdam) 371, 159 (2016).

[35] S. Kohler, T. Dittrich, and P. Hänggi, Floquet-Markovian description of the parametrically driven, dissipative harmonic quantum oscillator, Phys. Rev. E 55, 300 (1997).

[36] R. Graham and R. Hübner, Generalized quasi-energies and Floquet states for a dissipative systems, Ann. Phys. (N.Y.) 234, 300 (1994).

[37] R. Blattmann, P. Hänggi, and S. Kohler, Qubit interference at avoided crossings: The role of driving shape and bath coupling, Phys. Rev. A 91, 042109 (2015).

[38] See Supplemental Material at http://link.aps.org/ supplemental/10.1103/PhysRevLett.123.180402 for the derivation of the master equation and various technical details, which includes Refs. [17,30-36,39-42].

[39] Z. Su, A. B. Tacla, M. Hocevar, D. Car, S. R. Plissard, E. P. A. M. Bakkers, A. J. Daley, D. Pekker, and S. M. Frolov, Andreev molecules in semiconductor nanowire double quantum dots, Nat. Commun. 8, 585 (2017).

[40] M. Grifoni and P. Hänggi, Driven quantum tunneling, Phys. Rep. 304, 229 (1998).

[41] V. I. Yudin, A. V. Taichenachev, and M. Y. Basalaev, Dynamic steady state of periodically driven quantum systems, Phys. Rev. A 93, 013820 (2016).

[42] D. Malz and A. Nunnenkamp, Floquet approach to bichromatically driven cavity optomechanical systems, Phys. Rev. A 94, 023803 (2016).

[43] R. Delagrange, R. Weil, A. Kasumov, M. Ferrier, H. Bouchiat, and R. Deblock, $0-\pi$ Quantum transition in a carbon nanotube Josephson junction: Universal phase dependence and orbital degeneracy, Physica (Amsterdam) 536B, 211 (2018).

[44] T. Gericke, P. Wurtz, D. Reitz, T. Langen, and H. Ott, Highresolution scanning electron microscopy of an ultracold quantum gas, Nat. Phys. 4, 949 (2008).

[45] C. Weitenberg, M. Endres, J. F. Sherson, M. Cheneau, P. Schausz, T. Fukuhara, I. Bloch, and S. Kuhr, Single-spin addressing in an atomic Mott insulator, Nature (London) 471, 319 (2011).

[46] W. S. Bakr, J. I. Gillen, A. Peng, S. Folling, and M. Greiner, A quantum gas microscope for detecting single atoms in a
Hubbard-regime optical lattice, Nature (London) 462, 74 (2009).

[47] J. F. Sherson, C. Weitenberg, M. Endres, M. Cheneau, I. Bloch, and S. Kuhr, Single-atom-resolved fluorescence imaging of an atomic Mott insulator, Nature (London) 467, 68 (2010).

[48] H. P. Lüschen, P. Bordia, S. S. Hodgman, M. Schreiber, S. Sarkar, A. J. Daley, M. H. Fischer, E. Altman, I. Bloch, and U. Schneider, Signatures of Many-Body Localization in a Controlled Open Quantum System, Phys. Rev. X 7, 011034 (2017).

[49] S. Sarkar, S. Langer, J. Schachenmayer, and A. J. Daley, Light scattering and dissipative dynamics of many fermionic atoms in an optical lattice, Phys. Rev. A 90, 023618 (2014).

[50] H. Pichler, A.J. Daley, and P. Zoller, Nonequilibrium dynamics of bosonic atoms in optical lattices: Decoherence of many-body states due to spontaneous emission, Phys. Rev. A 82, 063605 (2010).

[51] E. P. L. van Nieuwenburg, J. Y. Malo, A. J. Daley, and M. H. Fischer, Dynamics of many-body localization in the presence of particle loss, Quantum Sci. Technol. 3, 01LT02 (2017).

[52] Z. Lenarcic and T. Prosen, Exact asymptotics of the current in boundary-driven dissipative quantum chains in large external fields, Phys. Rev. E 91, 030103(R) (2015).

[53] G. T. Landi, E. Novais, M. J. de Oliveira, and D. Karevski, Flux rectification in the quantum $X X Z$ chain, Phys. Rev. E 90, 042142 (2014).

[54] L. Schuab, E. Pereira, and G. T. Landi, Energy rectification in quantum graded spin chains: Analysis of the $X X Z$ model, Phys. Rev. E 94, 042122 (2016).

[55] E. Pereira, Rectification and one-way street for the energy current in boundary-driven asymmetric quantum spin chains, Phys. Rev. E 95, 030104(R) (2017).

[56] E. Pereira, Requisite ingredients for thermal rectification, Phys. Rev. E 96, 012114 (2017).

[57] V. Balachandran, G. Benenti, E. Pereira, G. Casati, and D. Poletti, Perfect Diode in Quantum Spin Chains, Phys. Rev. Lett. 120, 200603 (2018).

[58] E. Pereira, Heat, work, and energy currents in the boundarydriven $X X Z$ spin chain, Phys. Rev. E 97, 022115 (2018).

[59] T. Werlang, M. A. Marchiori, M. F. Cornelio, and D. Valente, Optimal rectification in the ultrastrong coupling regime, Phys. Rev. E 89, 062109 (2014).

[60] E. Mascarenhas, F. Damanet, S. Flannigan, L. Tagliacozzo, A. J. Daley, J. Goold, and I. de Vega, Nonreciprocal quantum transport at junctions of structured leads, Phys. Rev. B. 99, 245134 (2019).

[61] D. Malz and A. Nunnenkamp, Current rectification in a double quantum dot through fermionic reservoir engineering, Phys. Rev. B 97, 165308 (2018).

[62] K. Ono, D. G. Austing, Y. Tokura, and S. Tarucha, Current rectification by Pauli exclusion in a weakly coupled double quantum dot system, Science 297, 1313 (2002).

[63] A. C. Johnson, J. R. Petta, C. M. Marcus, M. P. Hanson, and A. C. Gossard, Singlet-triplet spin blockade and charge sensing in a few-electron double quantum dot, Phys. Rev. B 72, 165308 (2005). 
[64] G. Tang, L. Zhang, and J. Wang, Thermal rectification in a double quantum dots system with a polaron effect, Phys. Rev. B 97, 224311 (2018).

[65] R. Scheibner, M. König, D. Reuter, A. D. Wieck, C. Gould, H. Buhmannn, and L.W. Molenkamp, Quantum dot as thermal rectifier, New J. Phys. 10, 083016 (2008).

[66] A. Eichler, M. Weiss, S. Oberholzer, C. Schönenberger, A. Levy Yeyati, J. C. Cuevas, and A. Martín-Rodero, Even-Odd Effect in Andreev Transport through a Carbon Nanotube Quantum Dot, Phys. Rev. Lett. 99, 126602 (2007).

[67] S. Uchino, M. Ueda, and J.-P. Brantut, Universal noise in continuous transport measurements of interacting fermions, Phys. Rev. A 98, 063619 (2018).
[68] M. Benito and G. Platero, Floquet Majorana fermions in superconducting quantum dots, Physica (Amsterdam) 74E, 608 (2015).

[69] Y. Li, A. Kundu, F. Zhong, and B. Seradjeh, Tunable Floquet Majorana fermions in driven coupled quantum dots, Phys. Rev. B 90, 121401(R) (2014).

[70] R. Sánchez, B. Sothmann, A. N. Jordan, and M. Büttiker, Correlations of heat and charge currents in quantum-dot thermoelectric engines, New J. Phys. 15, 125001 (2013).

[71] J. P. Pekola, Towards quantum thermodynamics in electronic circuits, Nat. Phys. 11, 118 (2015).

[72] https://doi.org/10.15129/f8d30ceb-b1ea-45d8-9272-489de3629dfe. 